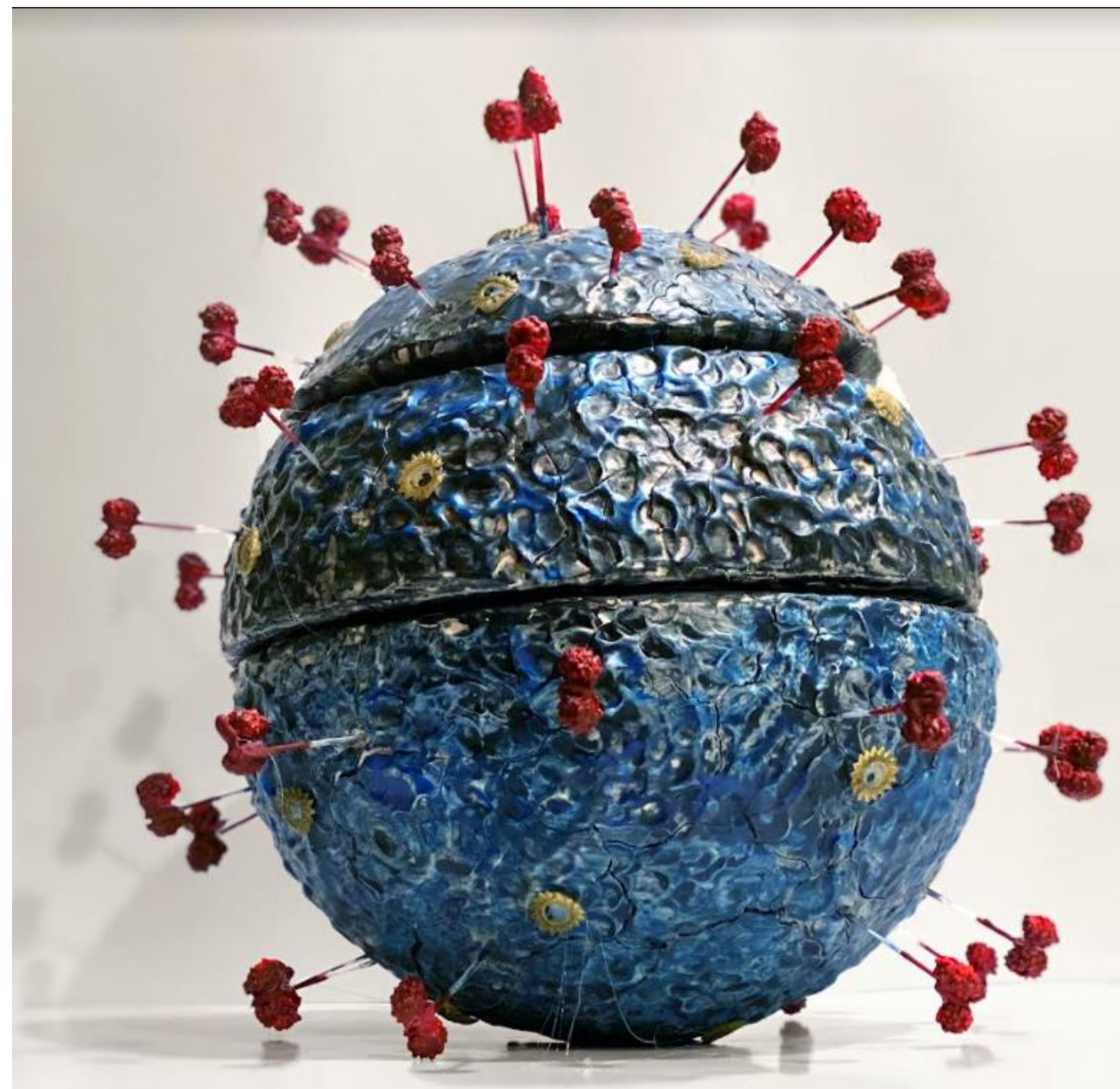

Title: Covid-19 - Assembled

Artist: Puja Rajesh

Medium: Candy peanuts, Styrofoam, clay, paint, plastic teaspoons, a plastic cup, LED lights, glue.

CPuja Rajesh (2021) Pur142@usask.ca Biomedical Foundations, College of Arts and Science, University of Saskatchewan

\title{
Artist's Statement
}

This piece is from my ART 141 class and is a light-up model of the coronavirus itself, able to be assembled and disassembled at will, ironically allowing people to influence the virus, and not the other way around. 


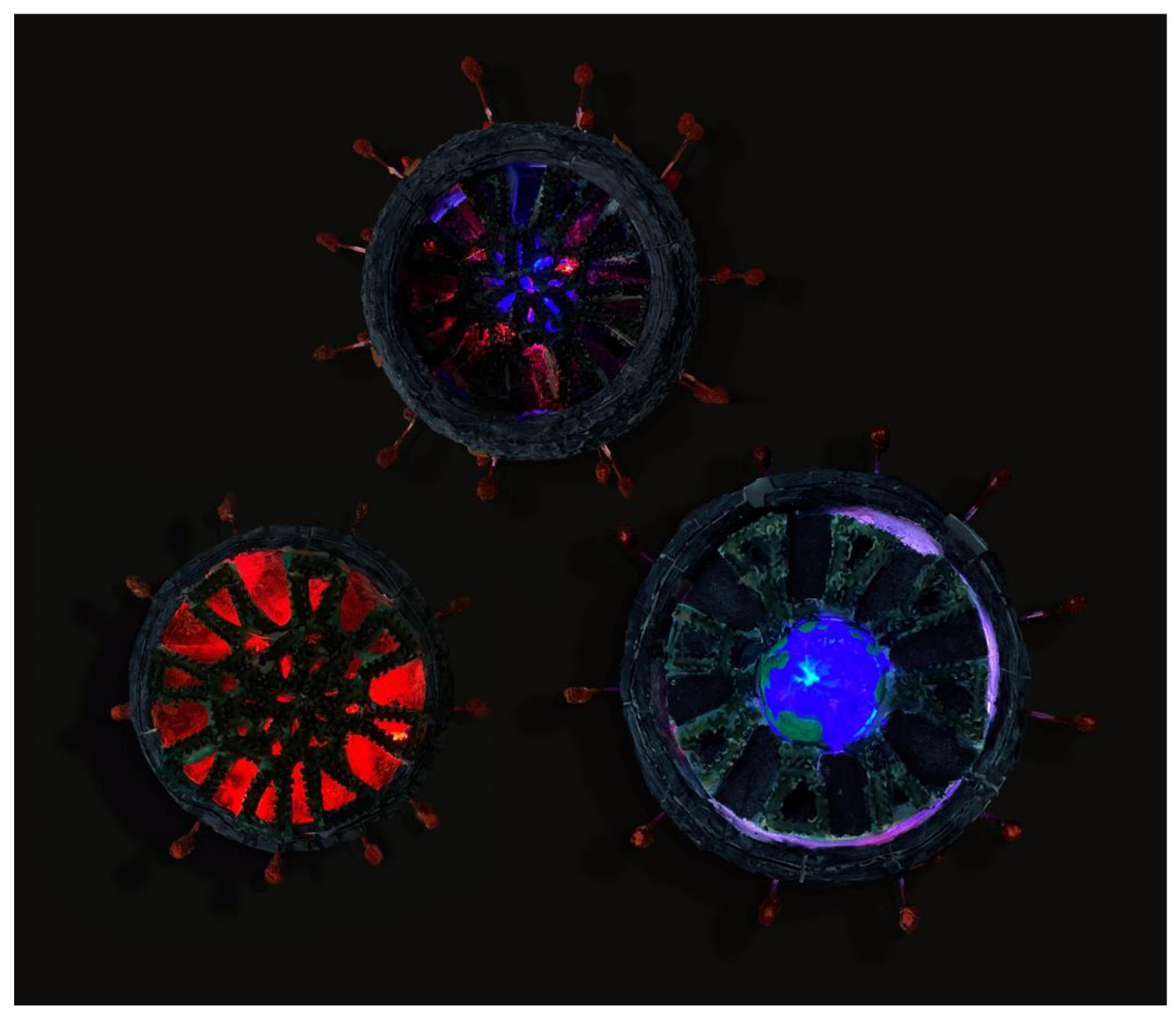

Title: Covid-19 - Disassembled

Artist: Puja Rajesh

Medium: Candy peanuts, Styrofoam, clay, paint, plastic teaspoons, a plastic cup, LED lights, glue.

CPuja Rajesh (2021) Pur142@usask.ca Biomedical Foundations, College of Arts and Science, University of Saskatchewan

\section{Artist's Statement}

This piece is from my ART 141 class and is a light-up model of the coronavirus itself, able to be assembled and disassembled at will, ironically allowing people to influence the virus, and not the other way around. 


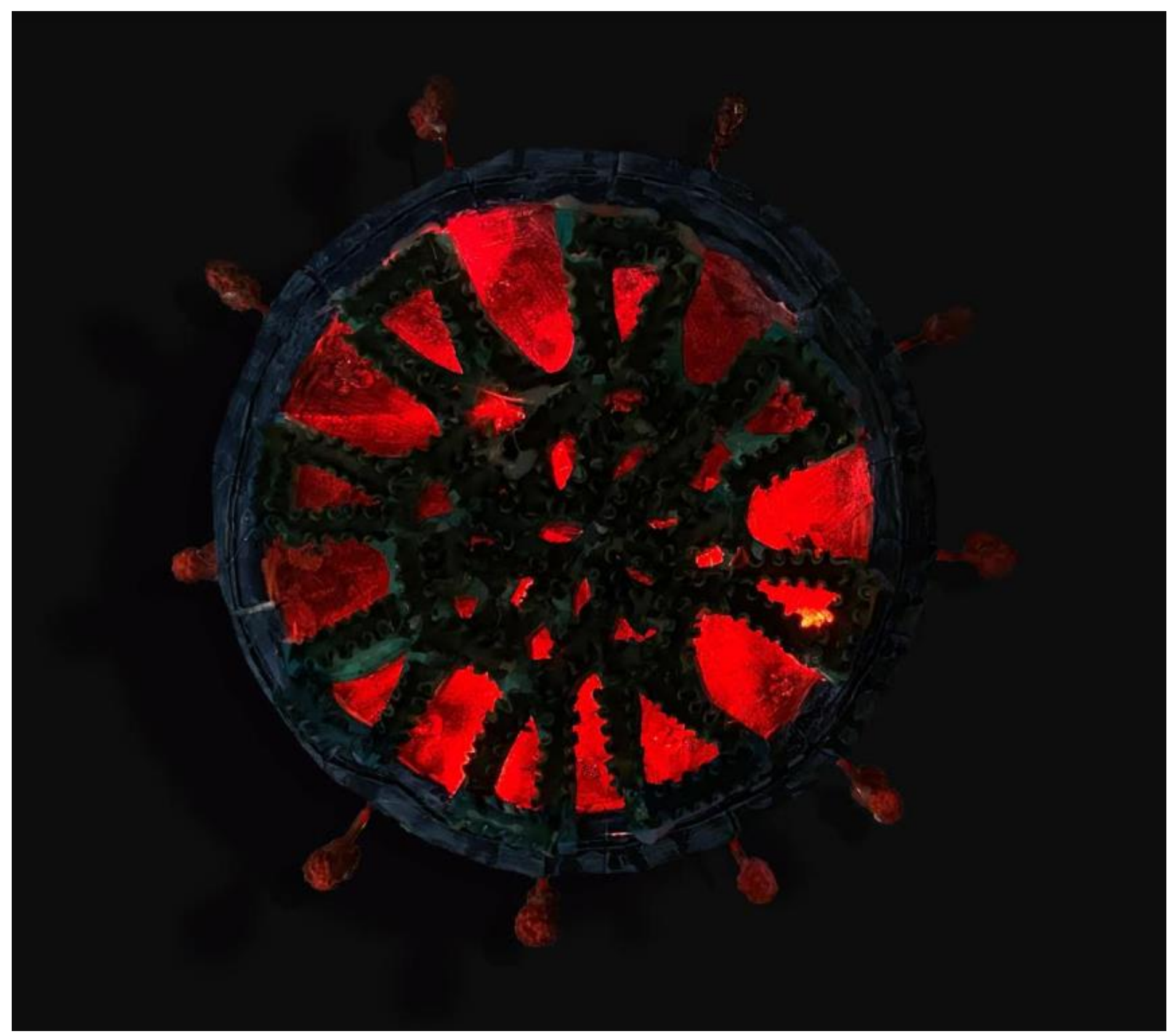

Title: Covid-19 - Disassembled (Detail)

Artist: Puja Rajesh

Medium: Candy peanuts, Styrofoam, clay, paint, plastic teaspoons, a plastic cup, LED lights, glue.

CPuja Rajesh (2021) Pur142@usask.ca Biomedical Foundations, College of Arts and Science, University of Saskatchewan

\section{Artist's Statement}

This piece is from my ART 141 class and is a light-up model of the coronavirus itself, able to be assembled and disassembled at will, ironically allowing people to influence the virus, and not the other way around. 